\title{
BMJ Open Use of complexity theory in health and social care: a scoping review protocol
}

\author{
Aine Carroll (D , ${ }^{1,2}$ Diarmuid Stokes, ${ }^{3}$ Andrew Darley ${ }^{1}$
}

To cite: Carroll A, Stokes D, Darley A. Use of complexity theory in health and social care: a scoping review protocol. BMJ Open 2021;11:e047633. doi:10.1136/ bmjopen-2020-047633

- Prepublication history and additional supplemental material for this paper are available online.To view these files, please visit the journal online (http://dx. doi.org/10.1136/bmjopen-2020047633).

Received 09 December 2020 Accepted 29 June 2021

Check for updates

(C) Author(s) (or their employer(s)) 2021. Re-use permitted under CC BY-NC. No commercial re-use. See rights and permissions. Published by BMJ.

${ }^{1}$ School of Medicine, University College Dublin, Dublin, Ireland ${ }^{2}$ Medical Department, National Rehabilitation Hospital, Dublin, Ireland

${ }^{3}$ UCD Library, University College Dublin, Dublin, Ireland

Correspondence to

Professor Aine Carroll;

aine.carroll@ucd.ie

\begin{abstract}
Introduction Despite the use of a wide variety of improvement tools and approaches, healthcare organisations continue to struggle in several key areas. Complexity-informed approaches have the potential to offer health and social care a new paradigm for understanding, designing, implementing and evaluating solutions, yet so far has failed to gain the traction anticipated some years ago. There is a growing need for high quality syntheses of the existing knowledge base in this area and given the diversity of theory and approaches, a scoping review is the best approach to curate this knowledge.
\end{abstract}

Methods A scoping review of relevant literature from January 2000 to present, using the refined Arksey and O'Malley six-stage framework will be conducted. This protocol will follow the Preferred Reporting Items for Systematic Reviews and Meta-analysis Protocols Extension for Scoping Reviews. A three-step search strategy will be used. An initial search of databases will be undertaken to identify key search terms followed by an analysis of retrieved papers title and abstract text words, and of index terms used to describe the articles. A second search using all identified keywords and index terms will then be undertaken across all included databases. Third, the reference lists of identified reports and articles will be searched. Authors of primary articles will be contacted and a search for grey material performed. Finally, a complete search strategy of one major database will be included. Ethics and dissemination As this is a scoping review, ethical approval is not required. The results of the scoping review will be published in a peer-reviewed journal and presented at national and international conferences and will guide a large research project investigating teamwork. All data will be stored in accordance with best General Data Protection Regulation practice.

Registration This scoping review protocol has been registered with Open Science Framework.

\section{BACKGROUND}

Across the globe, health and social care organisations are struggling in several key areas; inequity of access, service fragmentation, suboptimal care quality, system inefficiencies, unaffordability and complexity. ${ }^{1-3}$ In a desire to address some of these issues, in 2015, the United Nations (UN) launched the sustainable development goals, a universal call to action to end poverty, protect the planet and improve the lives and prospects of everyone,
Strengths and limitations of this study

- This study will provide a comprehensive synthesis of the literature relating to complexity and health and social care research.

- The review will follow a validated framework.

- As it is a scoping review, the quality of evidence will not be assessed.

- Only English language studies will be included.

everywhere. The 17 goals were adopted by all UN member states as part of the 2030 Agenda for Sustainable Development, which set out a 15-year plan to achieve the goals. ${ }^{4}$ It has long been recognised that in order to adequately address the shared challenges of ageing populations, chronic diseases and the social determinants of health will require a fundamental shift in how health and social care is imagined, designed and delivered. ${ }^{2}$ There have been a great many attempts at improving health and social care services over the last 50 years using techniques mainly adapted from industrial settings, yet these efforts do not seem to have resulted in any meaningful and sustained improvement. ${ }^{5-7}$ Health and social care have changed dramatically over those years. Life expectancy has increased, and our citizens are living with the consequences of diseases from which previous generations would have died. This necessitates a radical change in how we plan and deliver health and social care, yet our health and social care models are still predominantly hospital-orientated, episodic and curative in nature. A shift to continuity of care in the community across the continuum of care from primary prevention to end of life care that is coordinated and integrated is required. There have been many national and international mandates in health and social care reform that demand more integrated services across disciplines, organisations and domains. People-centred and integrated health services have been shown to generate benefits for people and health systems in countries across the world. ${ }^{8}$ The evidence suggests that people-centred and 
integrated services are essential components of building universal health coverage and can improve health status. ${ }^{3}$ How best to effect integrated care unfortunately remains an enigma; How should countries imagine, design and deliver integrated care? What models and philosophies are best suited to reimagining health and social care provision? What new theories are emerging that might inform this shift?

Such a paradigm shift in thinking is starting to emerge with the advent of complex systems thinking. Complexity science has emerged from diverse academic disciplines as a way to understand the world in which we live. It has been used to examine the unpredictable, disorderly, non-linear and uncontrollable ways in which living systems behave. Johnson offered a description of complexity as 'the study of phenomena which emerge from a collection of interacting objects ${ }^{9}$ (pg 1). Complexity science is a useful framework for studying dynamic, real-life systems ${ }^{10} 11$ and enables individuals to explore the messy unpredictable behaviour of diverse, interconnected agents and processes from a systems perspective (a system being a set of elements standing in interrelation). ${ }^{12}$ Modern health and social care are increasingly recognised as complex and therefore needs to be viewed through a complexity lens when researching and developing solutions rather than the more traditional positivistic linear reductionist approach. ${ }^{13-18}$ The emerging science of complexity has the potential to offer health and social care professionals a new approach for understanding, designing and implementing solutions in these often chaotic and complex times, yet so far has failed to gain the traction anticipated some years ago. Despite the increasing adoption of complexity-informed approaches in health and social care, Greenhalgh and Papoutsi highlighted how complexity in empirical health services research is often conducted in name only' and lack the necessary engagement with the logic that underpins it ${ }^{19}$ (pg 1).

There have been a number of evidence syntheses examining complexity theory in healthcare and its evaluation. Thompson and colleagues performed a scoping review of complexity theory in health services research and concluded that although the use of complexity theory shows promise, conceptual confusion and inconsistent application hinders the operationalisation of this potentially important perspective. ${ }^{20}$ Rusoja et al carried out a systematic review of the key systems thinking and complexity ideas in health and found that there was a need for further study and practical application. ${ }^{17}$ Brainard and Hunter's scoping review found that it is not feasible to effectively research and evaluate the efficacy of complexity-informed health interventions due to the lack of recognition of complexity science in its design or evaluation process. ${ }^{21}$ Additionally, Braithwaite and colleagues have published a number of papers looking at various aspects of complexity in healthcare and acknowledged the challenge of describing complexity and complex adaptive systems and also underlined the need to delineate the characteristics. ${ }^{22-25}$
Our preliminary literature scan and discussions with stakeholders (including clinical and policy decision makers, as well as patients, caregivers and key authors) have identified knowledge gaps about how to best use complexity theory to inform the design, delivery and evaluation of health and social care services. In particular, the knowledge gaps that were discussed regarding complexityinformed health and social care research were the lack of clarity regarding the definition of complexity theory and its characteristics, the settings and professions in which it has been used, knowledge mobilisation and the impact of previous studies.

In this paper, we set out the protocol for a scoping review to examine the literature on complexity theory in health and social care research. After careful consideration of the best form of evidence synthesis and using the indications for performing reviews identified by Munn $e t a l,{ }^{26}$ we concluded that a scoping review was the most appropriate approach to enable the authors to comprehensively map the international literature from various evidence sources relating to how complexity theory has been applied in research in health and social care.

As is a requirement for other forms of evidence synthesis, it is advised that an a priori protocol should be developed before undertaking the scoping review and this paper presents that protocol. ${ }^{2627}$

Building on the evidence highlighted in previous reviews ${ }^{17} 202123$ and agreed on with stakeholders, the aim of this review is to address the existing ambiguity regarding the definition of and characteristics of how complexity theory is used and described in health and social care research. The authors aim to comprehensively understand the gaps in knowledge regarding the settings and professions in which complexity theory has been used, for health and social care research, as well as the knowledge mobilisation and impact resulting from empirical studies which adopted a complexity-informed approach. This scoping review is being undertaken to inform a research project that will investigate leadership in a complex system, but we believe the review will have broader appeal to clinicians, managers and researchers that wish to identify knowledge gaps and to see better outcomes for patients.

\section{METHODS AND ANALYSIS}

We will adopt the framework for scoping reviews developed by Arksey and O'Malley and refined by subsequent authors. ${ }^{28-32}$ This methodology consists of six stages: (1) specifying the research question, (2) identifying relevant studies, (3) study selection, (4) charting the data and reporting the results, (5) collating and summarising the findings and (6) consultation with key stakeholders.

This scoping review protocol follows the Preferred Reporting Items for Systematic Reviews and Meta-analysis Protocols Extension for Scoping Reviews (PRISMA-ScR). ${ }^{33}$ 


\section{Patient and public involvement}

This review is being performed to inform research on leading in complex times whereby patient engagement is a central component of the study. This protocol will be shared with the organisation's patient forum for review and feedback.

\section{Specifying the research question}

Prior to identifying the research question, an exploratory review of the literature on the use of complexity theory in health and social care helped refine the scope of this protocol. ${ }^{17202125}$ Key authors of these systematic reviews were contacted for their advice for the development of this protocol. Based on this initial research, the following research question emerged: How has complexity theory been used in health and social care research? In the light of this research question, the key objectives of the scoping review are:

- To map descriptions (including definitions and characteristics) of the use of complexity theory in health and social care research.

- To examine the methodologies and extent to which complexity theory has been applied in health and social care research.

- To examine the settings and professions studied using complexity theory.

- To investigate the impact of the application of complexity theory.

- To identify if research findings, conclusions and recommendations can provide evidence of knowledge/capacity building and change.

- To determine if there are any gaps in research and make recommendations for future research.

\section{Identifying relevant studies}

\section{Inclusion criteria}

Following the framework of Arksey and O'Malley, the second stage of the scoping review process will identify the inclusion criteria. ${ }^{32}$ The 'PCC' mnemonic is used a guide to frame the research question and the related search, that is, population, concept and context. ${ }^{31}$ In the current review, this refers to:

- Types of participants: this scoping review will consider health and social care professionals involved in empirical research-nursing, medical, allied healthcare professionals/health and social care professionals, health and social care management.

- Concept: this scoping review will consider studies which use complexity theory in health and social care settings.

- Context: this scoping review will consider studies using complexity theory, that were carried out in health and social care settings including secondary care, primary care, rehabilitation and community settings, including non-governmental organisations.

- Types of evidence sources: this scoping review will consider both qualitative and quantitative primary research in the English language between the years
2000 and 2021 that illustrate the use of complexity theory informed approaches to research, the research process and the research findings. This timespan was determined by the authors to be appropriate for the search given the emerging trend of complexity science within health and social care in the early 2000s. This will include systematic reviews, meta-analyses, scoping reviews, evidence maps, rapid reviews, literature reviews, evidence syntheses, narrative reviews and critical reviews. Primary research studies contained in relevant reviews will be extracted and included in the screening process to ensure comprehensiveness. Unpublished (grey literature) will also be included such as presentations, reports and dissertations. Grey literature will be sourced from a range of health and social care-related evidence sources including OpenGrey, Lenus, Google and Google Scholar. It was agreed to exclude conference abstracts, book reviews, commentaries or editorial articles and non-English articles.

\section{Search strategy}

A three-step search strategy will be used in this review. An initial limited search of databases MEDLINE and CINAHL will be undertaken followed by an analysis of the text words contained in the title and abstract of retrieved papers, and of the index terms used to describe the articles.

The main search will be done by a research librarian and peer-reviewed by another librarian as recommended. ${ }^{32}$ The input of a research librarian during this stage will aid in designing and refining the search to ensure the entire search strategy and results are transparent, auditable and replicable in the future. The research librarian will advise on the most appropriate Medical Subject Headings terms for the PubMed search and how to modify them for the different databases used. Based on this exploratory scoping phase, the search strings for each database will be finalised. The search terms are listed in the online supplemental file 1. Boolean operators 'AND/OR' will be used to construct the search strategy and use of 'Truncation*' will also reveal related terms.

A second search using all identified keywords and index terms will then be undertaken across all included databases. Databases to be searched include Cochrane Database of Systematic Reviews, MEDLINE, CINAHL, EMBASE, Web of Science, PSYCHINFO, The NHS Economic Evaluation Database, The Health Economic Evaluations Database.

Third, the reference lists of identified reports and articles will be searched for additional sources. Reviewers intend to contact authors of primary articles or reviews for further information if this is relevant. A search for grey material, such as unpublished work, conferences, reports, website information, may also be necessary. 


\section{Source of evidence selection}

Selection of evidence will be performed based on the inclusion criteria pre-specified already in the review protocol. Source selection (both at title/abstract screening and full-text screening) will be performed by two reviewers, independently. Disagreements between reviewers will be solved by consensus or by the decision of a third reviewer. Articles will be retrieved from each database and imported into the reference management software Endnote, a bibliographic manager. Systematic de-duplication will be performed using the Bramer method for Endnote users to ensure each study retrieved is not repeated, providing a more accurate final number count. ${ }^{34}$ The systematic review software tool, Covidence ( www.covidence.org), will be used to conduct the screening of the retrieved literature. This software ensures that each article is decided as included or excluded by two reviewers independently and reduces screening bias. Any disagreements will be resolved by consensus or involvement of a third reviewer. A pilot test of the screening process using Covidence will be undertaken, featuring a random sample of the title/abstracts found $(n=25)$. This process is to ensure a consistent methodology among all reviewers before undertaking the screening of all identified literature. The process of study selection will be reported using a PRISMA flow chart, which will then be updated once the review is completed. ${ }^{33}$

\section{Data extraction/'charting'}

A data extraction framework guided by our research question will be developed and piloted at the protocol stage to record the key information of each source, such as author, reference and results or findings relevant to the review question/s. This may be further refined at the review stage and the charting table will be updated accordingly. Key information will include:

- Study descriptors including:

- Author(s).

- Year of publication.

- Origin/country of origin (where the source was published or conducted).

- Research design and methodology.

- Ethical considerations of the study.

- Professionals involved.

- Research setting.

- Interprofessional focus.

- Aims/purpose of research.

- Attributes/characteristics of complexity theory used.

- Definition/description of complexity theory used.

- How complexity theory was used.

- Knowledge mobilisation that is, all activities undertaken to disseminate findings.

- Impact of research.

- Key findings that relate to the scoping review question.

- Author bibliometrics.

The extraction framework will be pilot tested by two team members on a sample of the included studies $(10 \%$ of the complete list of retrieved studies) to ensure that the coding framework is consistently applied. The categories may be modified and the data extraction framework will be revised if necessary. Emerging questions within the team when piloting the framework will be discussed and any disagreement will be resolved through team consultation. This pilot step will ensure that the authors are transparent and clear in their methods regarding what and how they will extract data.

\section{Collating, summarising and reporting the results}

The analysis of the data collected using the data extraction framework will provide information on the body of research where complexity theory has been applied in health and social care research. It will be possible to highlight the effectiveness of complexity theory in this context as the review focuses on evidence of knowledge mobilisation and impact of the research. Conversely, it will also show areas that have been under-researched and may require further investigation.

The results will be presented in an aggregate and visual form (eg, using tables and charts as appropriate) and in a descriptive format that aligns with the objectives and scope of the review.

The results summary will describe the aims or purposes of the reviewed sources, the methodologies applied and results that relate to the review questions.

The results will be classified under main conceptual categories that will be obtained during the results extraction. For each category, a clear explanation will be provided.

\section{Expert consultation}

Levac and colleagues suggested that consultation is necessary to provide insight and input beyond the literature. ${ }^{30}$ Consistent with a participatory approach to research, the authors engaged several relevant stakeholder groups (ie, researchers, patients, health and social care professionals and policy makers) to inform the research question of the review. The purpose of this expert consultation was to identify stakeholder priorities and questions to guide the scoping review. They were consulted about their perceptions and/or experience of conducting research in the area of complexity in health and social care with a focus on what they considered to be important for future work. Consultations were conducted individually, either in-person via telephone or email exchange and notes were recorded to capture the data and analysed by the lead author (AC). Individuals who were not familiar with research practices or the field of complexity were invited to an information session and provided with written materials to inform them before engaging in the consultation. These stakeholders will be further consulted during the undertaking of the review. The stakeholder groups will be invited to provide further feedback on the revised scoping review criteria once published. The author team will also disseminate initial findings and interpretations of the identified evidence to stakeholders in order to 
sense make together and capture their perspective of the preliminary results.

\section{Risk of bias assessment or quality appraisal}

Given that this scoping review has the aim of mapping all available evidence, we will not conduct any risk of bias assessment or quality appraisal of included studies. The authors seek to establish the breath of evidence of complexity theory in health and social care settings rather than its rigour and robustness. This approach is consistent with the methods manual published by the Joanna Briggs Institute ${ }^{27}$ as well as a database of scoping reviews on health-related topics.

\section{Dissemination}

This review will guide a large research project investigating teamwork in a tertiary hospital during a period of change in Ireland. The results of the scoping review will be published in a peer-reviewed journal and presented at local, regional, national and international conferences. All data will be stored in accordance with best General Data Protection Regulation practice.

\section{Twitter Andrew Darley @adarleyresearch}

Contributors AC came up with the original concept for the review and wrote the original draft protocol. $A D$ and $D S$ made substantial contributions to the search strategy and design and writing of subsequent versions and both revised the first draft critically for important intellectual content. All three authors approved the final version for submission.

Funding The authors have not declared a specific grant for this research from any funding agency in the public, commercial or not-for-profit sectors.

Competing interests None declared.

Patient consent for publication Not required.

Provenance and peer review Not commissioned; externally peer reviewed.

Supplemental material This content has been supplied by the author(s). It has not been vetted by BMJ Publishing Group Limited (BMJ) and may not have been peer-reviewed. Any opinions or recommendations discussed are solely those of the author(s) and are not endorsed by BMJ. BMJ disclaims all liability and responsibility arising from any reliance placed on the content. Where the content includes any translated material, BMJ does not warrant the accuracy and reliability of the translations (including but not limited to local regulations, clinical guidelines, terminology, drug names and drug dosages), and is not responsible for any error and/or omissions arising from translation and adaptation or otherwise.

Open access This is an open access article distributed in accordance with the Creative Commons Attribution Non Commercial (CC BY-NC 4.0) license, which permits others to distribute, remix, adapt, build upon this work non-commercially, and license their derivative works on different terms, provided the original work is properly cited, appropriate credit is given, any changes made indicated, and the use is non-commercial. See: http://creativecommons.org/licenses/by-nc/4.0/.

ORCID iD

Aine Carroll http://orcid.org/0000-0002-4383-8650

\section{REFERENCES}

1 Plsek PE, Wilson T. Complexity, leadership, and management in healthcare organisations. BMJ 2001;323:746-9.

2 Kruk ME, Gage AD, Arsenault C, et al. High-Quality health systems in the sustainable development goals era: time for a revolution. Lancet Glob Health 2018;6:e1196-252.

3 WHO. Framework on integrated people-centred health services: report by the Secretariat 2016, 2019.
4 United Nations General Assembly. Transforming our world: the 2030 agenda for sustainable development: draft resolution referred to the United nations Summit for the adoption of the post-2015 development agenda by the general assembly at its sixty-ninth session. United nations, 2015

5 Dixon-Woods M. How to improve healthcare improvement-an essay by Mary Dixon-Woods. BMJ 2019;367:I5514.

6 Dixon-Woods M, Martin GP. Does quality improvement improve quality? Future Hosp J 2016;3:191-4.

7 Walshe K. Pseudoinnovation: the development and spread of healthcare quality improvement methodologies. Int J Qual Health Care 2009;21:153-9.

8 Robertson H. Integration of health and social care a review of literature and models implications for Scotland. Royal College of nursing Scotland, 2011: 1-42.

9 Johnson N. Simply complexity: a clear guide to complexity theory. Oneworld Publications, 2009.

10 Arthur WB. Complexity and the economy. Science 1999;284:107-9.

11 Boulton JG, Allen PM, Bowman C. Embracing complexity: strategic perspectives for an age of turbulence. OUP Oxford, 2015.

12 Simon HA. The sciences of the artificial. MIT press, 2019.

13 Burns JP. Complexity science and leadership in healthcare. J Nurs Adm 2001;31:474-82.

14 Fraser SW, Greenhalgh T. Complexity science: coping with complexity: educating for capability. BMJ 2001;323:799-803.

15 Hanson WR, Ford R. Complexity leadership in healthcare: leader network awareness. Procedia Soc Behav Sci 2010;2:6587-96.

16 Plsek PE, Greenhalgh T. The challenge of complexity in health care. Bmj 2001;323:625-8.

17 Rusoja E, Haynie D, Sievers J, et al. Thinking about complexity in health: a systematic review of the key systems thinking and complexity ideas in health. J Eval Clin Pract 2018;24:600-6.

18 Carroll Á. The Irish healthcare system as a complex adaptive system. Ir Med J 2021;114:332.

19 Greenhalgh T, Papoutsi C. Studying complexity in health services research: desperately seeking an overdue paradigm shift. BMC Med 2018;16:95-6.

20 Thompson DS, Fazio X, Kustra E, et al. Scoping review of complexity theory in health services research. BMC Health Serv Res 2016;16:87.

21 Brainard J, Hunter PR. Do complexity-informed health interventions work? A scoping review. Implement Sci 2016;11:127.

22 Braithwaite J. Changing how we think about healthcare improvement. BMJ 2018;361:k2014.

23 Braithwaite J, Churruca K, Ellis LA. Complexity science in healthcare. Sydney: Australian Institute of Health Innovation, Macquarie University, 2017.

24 Braithwaite J, Churruca K, Long JC, et al. When complexity science meets implementation science: a theoretical and empirical analysis of systems change. BMC Med 2018;16:63-14.

25 Churruca K, Pomare C, Ellis LA, et al. The influence of complexity: a bibliometric analysis of complexity science in healthcare. BMJ Open 2019;9:e027308.

26 Munn Z, Peters MDJ, Stern C, et al. Systematic review or scoping review? guidance for authors when choosing between a systematic or scoping review approach. BMC Med Res Methodol 2018;18:143.

27 Peters M, Godfrey CM, Mclnerney P, et al. The Joanna Briggs Institute reviewers' manual 2015: methodology for JBI scoping reviews, 2015.

28 Anderson S, Allen P, Peckham S, et al. Asking the right questions: Scoping studies in the commissioning of research on the organisation and delivery of health services. Health Res Policy Syst 2008;6:1-12.

29 Daudt HML, van Mossel C, Scott SJ. Enhancing the scoping study methodology: a large, inter-professional team's experience with Arksey and O'Malley's framework. BMC Med Res Methodol 2013;13:48.

30 Levac D, Colquhoun H, O'Brien KK. Scoping studies: advancing the methodology. Implement Sci 2010;5:69.

31 Aromataris E, Munn Z. JBI manual for evidence synthesis. Adelaide: The Joanna Briggs Institute, 2020.

32 Arksey H, O'Malley L. Scoping studies: towards a methodological framework. Int J Soc Res Methodol 2005;8:19-32.

33 Tricco AC, Lillie E, Zarin W, et al. PRISMA extension for scoping reviews (PRISMA-ScR): checklist and explanation. Ann Intern Med 2018;169:467-73.

34 Bramer WM, Giustini D, de Jonge GB, et al. De-duplication of database search results for systematic reviews in endnote. $J$ Med Libr Assoc 2016;104:240-3. 